\title{
La peau : un organe lymphoïde périphérique
}

La peau est le témoin et l'acteur de nombreuses réactions immunologiques. Elle possède deux types de cellules immunitaires, les cellules de Langerhans qui présentent l'antigène aux lymphocytes, et les cellules dendritiques $\mathrm{Thy}_{-1} \mathrm{I}^{+}$qui pourraient être des lymphocytes $T$ appartenant à une population de différenciation très précoce, « pré-thymique », ressemblant à certaines cellules natural killer. L'étude de la stimulation des lymphocytes $\mathbf{T}$ du donneur de greffe par les cellules cutanées du receveur, effectuée par culture mixte lympho-épidermique, est la meilleure manière de prévoir la redoutable réaction du greffon contre l'hôte chez les malades ayant reçu une greffe de moelle.

\section{Martine Bagot \\ Chef de clinique-assistant}

\section{Louis Dubertret}

Professeur de médecine. Directeur de recherche de l'U. 312 de l'Inserm

\section{TIRÉS A PART}

M. Bagot: Inserm U. 312, laboratoire de dermatologie, hôpital Henri-Mondor, 94010 Créteil, France.

\section{ADRESSE}

M. Bagot, L. Dubertret : Inserm U. 312, laboratoire de dermatologie, hôpital Henri-Mondor, 94010 Créteil, France.

$\mathrm{m} / \mathrm{s} n^{\circ} 5$ vol. 4, mai 88

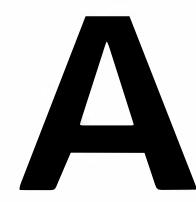

$\mathrm{u}$ cours des dernières années, plusieurs découvertes importantes ont permis de démontrer que la peau ne joue pas simplement un rôle de protection et de cible de maladies dont la physiopathologie fait intervenir des mécanismes immunologiques, mais représente un véritable organe immunocompétent doué de propriétés particulières. L'épiderme, qui est la première barrière entre l'organisme et le monde extérieur, possède tous les éléments nécessaires pour la constitution d'une réponse immınitaire. Il comprend plusieurs populations cellulaires bien différentes sur les plans morphologique et fonctionnel.

\section{Les cellules de Langerhans}

Les cellules de Langerhans représentent 3 à $5 \%$ des cellules épidermiques. Elles ont été décrites dès 1868 par Paul Langerhans, mais leur fonction immunologique n'a été reconnue qu'au cours des 15 dernières années. Originaires de la moelle osseuse, elles sont caractéri- sées par leur morphologie dendritique et la présence d'un organite cytoplasmique visible en microscopie électronique appelé granule de Birbeck (figure 1, page 312) [1]. Ces cellules ont des récepteurs pour le frag. ment FC des immunoglobulines et la fraction C3b du complément [2]. Elles expriment l'antigène T6 (CDI) ce qui permet de les identifier facilement au sein des cellules épidermiques (figure 2, page 312) [3]. Lorsque les cellules de Langerhans sont incubées avec un anticorps anti-T6, puis un anticorps anti-immunoglobulines marqué à l'or, on peut mettre en évidence une internalisation des antigènes $\mathrm{CDl}$ et l'apparition de granules de Birbeck marqués [4]. Il est également possible d'observer la cointernalisation de molécules $\mathrm{CDl}$ et d'antigènes de classe II (HLA. DR) [5]. On pense donc que ces granules sont liés à des phénomènes d'endocytose. Les cellules de Langerhans expriment des quantités variables d'antigène CD4 [6]. Elles peuvent donc être infectées par le virus HIV, et leur nombre est diminué dans l'épiderme de patients atteints 


\section{RÉFÉRENCES}

l. Birbeck MS, Breathnach AS, Everall JD An electron microscope study of basal melanocytes and high-level clear cells (Langerhans cells) in vitiligo. J Invest Dermatol 1961 ; $37: 51-63$.

2. Stingl G, Tamaki K, Katz SI. Origin and function of epidermal Langerhans cells. Immunol Rev $1980 ; 53$ : 149-74.

3. Fithian E, Kung P, Goldstein G, Rubenfeld M, Fenoglio C, Edelson R. Reactivity of Langerhans cells with hybridoma antibody. Proc Natl Acad Sci USA 1981 ; 78 : 2541-4.

4. Hanau D, Fabre M, Schmitt DA, et al. Human epidermal Langerhans cells internalize by receptor-mediated endocytosis T6 (CDI «NAl/34») surface antigen. Birbeck granules are involved in the intracellular traffic of the T6 antigen. J Invest Dermatol 1987 ; 89: 172-7.

5. Hanau D, Fabre M, Schmitt DA, et al. Human epidermal Langerhans cells cointernalize by receptor-mediated endocytosis « non classical » major histocompatibility complex class I molecules (T6 antigens) and class II molecules (HLA-DR antigens). Proc Natl Acad Sci USA 1987; 84 : 2901-5.

6. Wood GS, Warner NL, Warnke RA. AntiLeu-3,'T4 antibodies react with cells of monocyte/macrophage and Langerhans lineage. J Immunol 1983 ; 131 : 212-216.

7. Rowden G. Lewis MG, Sullivan AK. Ia antigen expression on human epidermal Langerhans cells. Nature 1977 ; $268: 247-8$

8. Caughman SW, Sharrow SO, Shimada S et al. $\mathrm{Ia}^{+}$murine epidermal Langerhans cells are deficient in surface expression of the class I major histocompatibility complex Proc Natl Acad Sci USA 1986 ; 83 : 7438-42.

9. Steiner G, Tschachler E. Tani M, et al. Interleukin 2 receptors on cultured murine epidermal Langerhans cells. J Immunol 1986; 137 : 155-9.

10. Schuler G, Steinman RM. Murine epidermal Langerhans cells mature into potent immunostimulatory dendritic cells in vitro. Exp Med $1985 ; 161: 526-46$.

11. Steinman RM, Nussenzweig MC. Dendritic cells, features and functions. Immunol Rev $1980 ; 53: 127-74$.

12. Volc-Platzer B Majdic O, Knapp W, et al. Evidence of HLA-DR antigen biosynthesis by human keratinocytes in disease. $J$ Exp Med $1984 ; 159: 1784-9$

13. Niederwieser D, Auböck J, Troppmair J, et al. Interferon mediated induction of MHC antigen expression on human keratinocytes and its influence on in vitro alloimmune responses (sous presse).

14. Tschachler E, Schuler G, Hutterer J. Leibl H, Wolff K, Stingl G. Expression of Thy-l antigen by murine epidermal cells. $J$ Invest Dermatol $1983 ; 81$ : 282-5.

15. Bergstresser PR, Tigelaar RE, Dees JH Streilein JW. Thy-l antigen-bearing dendritic cells populate murine epidermis. I Invest

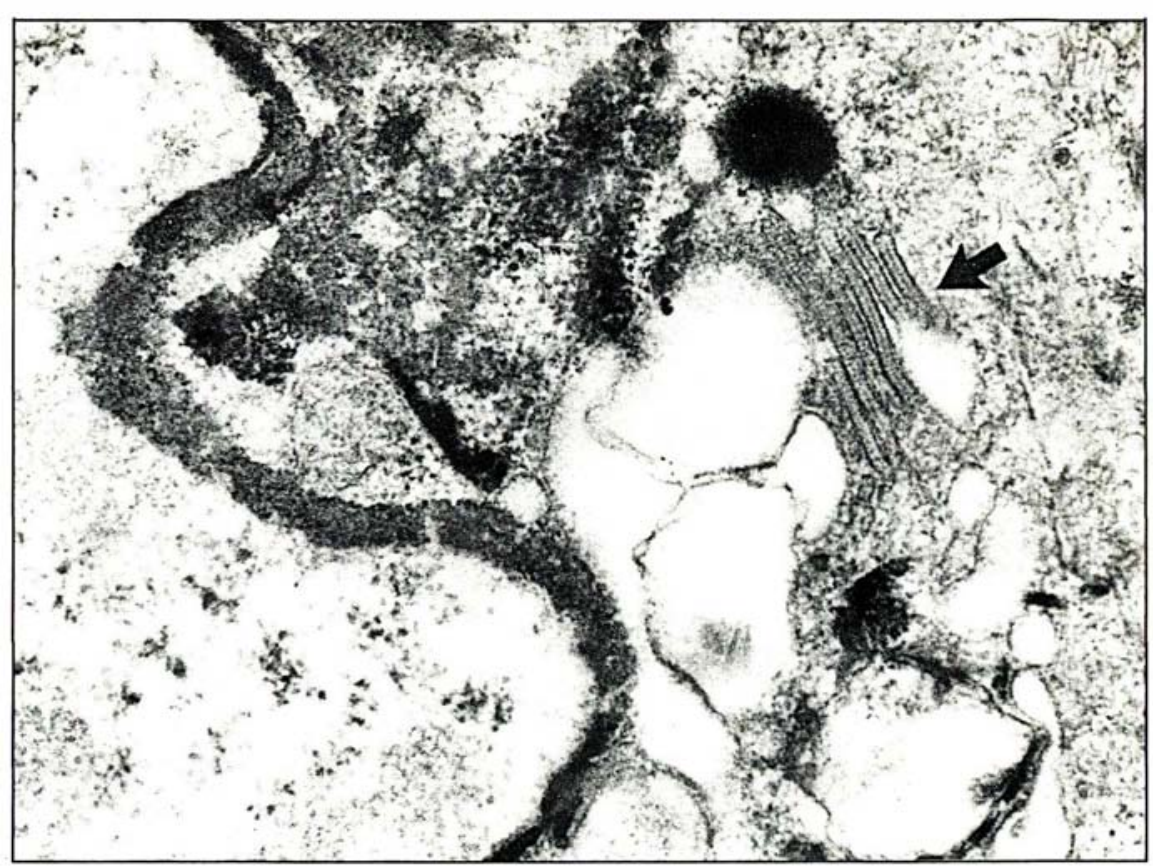

Figure 1. Granules de Birbeck visibles en microscopie électronique au sein d'une cellule de Langerhans. Ce cliché montre également l'activité peroxydasique endogène au niveau de l'enveloppe périnucléaire et du réticulum endoplasmique, révélée par incubation du tissu non fixé dans la diaminobenzidine $(\times 54000)$.

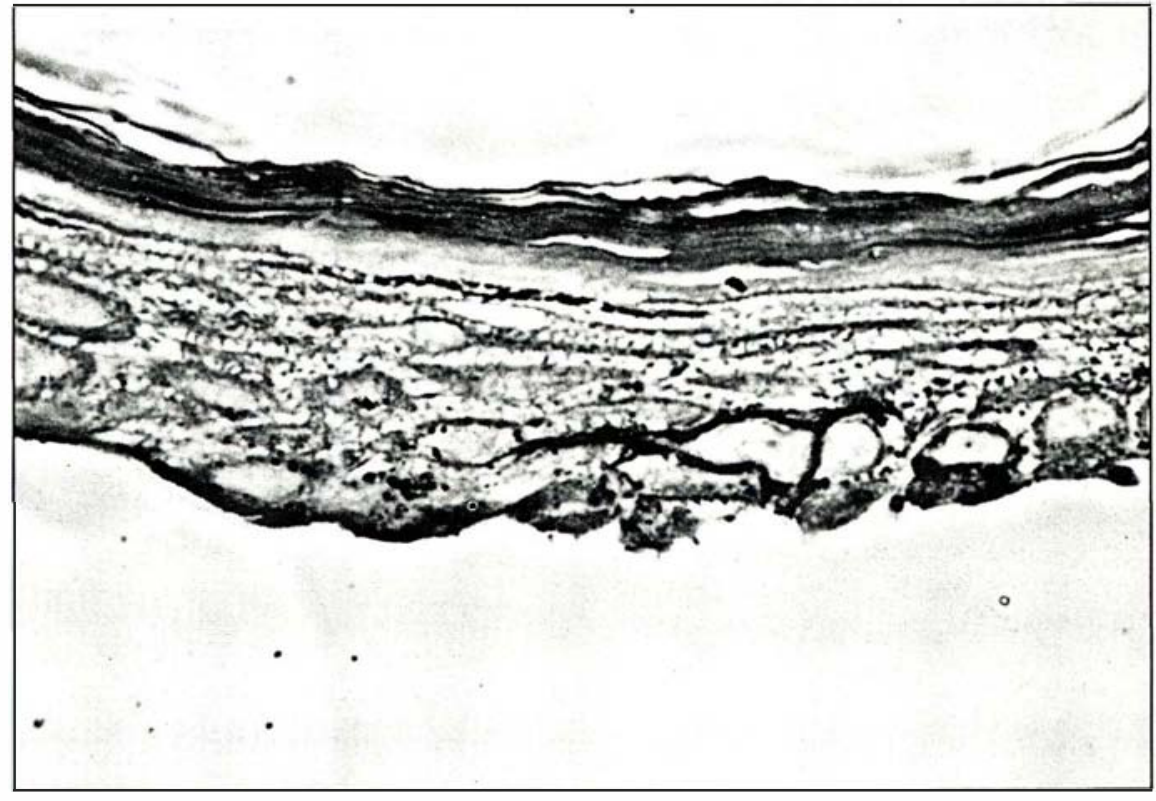

Figure 2. Cellule de Langerhans, avec ses prolongements dendritiques, révélée par technique d'immunoperoxydase anti-T6. Coupe semi-fine d'épaisseur $0,3 \mu m(\times 1500)$. 
de SIDA. Ce sont les seules cellules de l'épiderme qui expriment à l'état normal les produits de classe II du complexe majeur d'histocompatibilité $(\mathrm{CMH})[7]$. En revanche, elles n'expriment que des quantités très faibles d'antigènes de classe I[8]. Enfin, elles acquièrent en culture l'expression d'un récepteur de haute affinité pour l'interleukine 2 (IL2) [9] . La signification fonctionnelle de ces deux dernières observations est inconnue. Les cellules de Langerhans jouent un rôle majeur dans l'induction de l'hypersensibilité de contact et le rejet des greffes de peau. Il a été démontré qu'elles sont apparentées aux cellules dites « dendritiques », également retrouvées dans le sang et les ganglions [10]. Les cellules de cette lignée sont caractérisées par leur morphologie dendritique, l'expression de quantités importantes d'antigènes de classe II et de puissantes fonctions de présentation d'antigènes, particulièrement en réaction primaire [1 l]

\section{Les kératinocytes}

Les kératinocytes, qui représentent $95 \%$ des cellules épidermiques, permettent, à travers un programme de différenciation complexe, la formation d'une barrière imperméable, la couche cornée. A l'état normal, ces cellules n'expriment que les antigènes d'histocompatibilité de classe I et n'induisent aucune stimulation en réaction allogénique. En effet, une suspension de cellules épidermiques déplétée en cellules de Langerhans par incubation avec un anticorps anti HLA-DR et du complément, devient incapable d'induire la prolifération de lymphocytes allogéniques et d'engendrer une activité cytotoxique. L'apparition d'antigènes de classe II du CMH sur la membrane des kératinocytes a été rapportée dans de nombreuses maladies dermatologiques caractérisées par un infiltrat lymphocytaire dermique superficiel (réaction du greffon contre l'hôte, lichen, mycosis fongoïde, eczéma de contact) [12]. Le traitement in vitro des kératinocytes par l'interféron $\gamma$ induit également l'expression d'antigènes HLA-DR. Les kératinocytes ainsi traités par l'interféron $\gamma$ ne peuvent pas induire la prolifération de lymphocytes allogéniques en réac$\mathrm{m} / \mathrm{s} n^{\circ} 5 \mathrm{vol} .4$, mai 88 tion primaire ou secondaire, mais peuvent permettre l'entretien de lignées ou de clones lymphocytaires déjà établis in vitro [13]. Il est donc possible que ces cellules jouent un rôle d'amplification locale de réactions immunologiques initiées par les cellules de Langerhans.

Les mélanocytes et les cellules de Merkel, cellules épidermiques très minoritaires, n'ont pas de fonction immunologique connue. En revanche, une nouvelle population cellulaire épidermique a été récemment décrite: les cellules dendritiques Thy-1+.

\section{Cellules épidermiques dendritiques Thy-1+}

C'est en 1983 que deux équipes démontrent la présence, au niveau de l'épiderme de souris, de cellules exprimant des quantités importantes de l'antigène Thy-1, une glycoprotéine retrouvée essentiellement sur les lymphocytes $T$ et certaines cellules d'origine neuroectodermique [14, 15]. Ces cellules représentent 0,8 à $2,7 \%$ des cellules épidermiques. Leur densité est variable en fonction de la souche de souris et de la région du corps étudiée. Comme les cellules de Langerhans, elles ont une morphologie dendritique et sont originaires de la moelle osseuse [16, 17], mais contrairement aux cellules de Langerhans, elles n'expriment pas les antigènes de classe II du complexe majeur d'histocompatibilité $[14,15]$. Chez les chimères irradiées, les cellules dendritiques Thy- $\mathrm{I}^{+}$ recolonisent l'épiderme beaucoup plus lentement que les cellules de Langerhans. Les études phénotypiques ont montré que ces cellules sont différentes des lymphocytes $T$ matures, des lymphocytes B, des cellules de la lignée monocyte-macrophage et de la lignée dendritique [18]. Leur phénotype (Thy- $\mathrm{I}^{+}, \mathrm{Ly}-5^{+}$, asialo-GMl ${ }^{+}$, Ly-1 -, Lyt-2 -, L3T4 -, $\left.\mathrm{Ia}^{-}, \mathrm{sIg}^{-}\right)$est proche de celui de certaines cellules NK (natural killer)* $[19,21]$. Des travaux encore non publiés suggèrent que ces cellules produiraient des facteurs de crois-

* Voir lexique m/s no 6, vol. 3, p. 360. sance de type IL-2 et possèderaient une activité cytotoxique non restreinte par le complexe majeur d'histocompatibilité [22]. Il a été possible, en les cultivant en présence de lectines et d'IL-2, d'établir des lignées et des clones dérivés de ces cellules dendritiques, maintenant en culture pendant plusieurs mois le phénotype Thy- $\mathrm{I}^{+}, \mathrm{L} 3 \mathrm{~T} 4$-, Lyt-2 -. Ces lignées et ces clones, de même que les cellules in situ, expriment l'antigène T3 (ou CD3) [23]. Ils présentent des réarrangements des gènes codant pour la chaîne $\gamma$ du récepteur $T$ et expriment de grandes quantités d'ARNm $\gamma$ fonctionnel[24]. Leur membrane présente un hétérodimère associé au complexe CD3, constitué d'une chaîne $\gamma$ de 34 kilodaltons et d'une chaîne $\delta$ de 46 kilodaltons [25]. Les études biochimiques ont montré que ce récepteur est identique au récepteur $\gamma$ - $\delta$ retrouvé au niveau de sous-population très minoritaires de thymocytes et de lymphocytes T. Ces résultats suggèrent que les cellules épidermiques $\mathrm{Thy}^{-\mathrm{I}^{+}}$expriment ce récepteur in vivo, et pourraient être des lymphocytes $\mathrm{T}$ immatures. La mise en évidence de cellules dendritiques identiques dans l'épiderme de la souris nude fait émettre l'hypothèse que cette population représenterait des lymphocytes $T$ au stade pré-thymique et que l'épiderme pourrait être un site de différenciation extra-thymique [24]. Cette hypothèse est corroborée par les nombreuses similitudes retrouvées entre l'épithélium thymique et l'épiderme [26, 27].

Un dernier type cellulaire a été décrit dans l'épiderme de souris. Il s'agit de cellules qui, contrairement aux cellules de Langerhans, sont résistantes aux rayons ultraviolets, $\mathrm{Ia}^{-}$et $\mathrm{I}-\mathrm{J}^{+}$, et qui pourraient jouer un rôle dans l'activation de circuits suppresseurs [28].

\section{Production d'interleukines}

Les cellules épidermiques synthétisent de grandes quantités d'interleukines. On peut citer l'epidermal cellderived thymocyte-activating factor ou ETAF, identique à l'IL-1 [29], un facteur proche de la thymopoïétine [27], un facteur de croissance des lymphocytes distinct de l'IL-2: le 


\section{RÉFÉRENCES}

16. Breathnach SM, Kate SI. Thy-1+ dendritic cells in murine epidermis are bone marrow derived. J Invest Dermatol 1984 ; 83 : 74-7.

17. Bergstresser PR, Tigelaar RE, Streilein JW. Thy-l antigen-bearing dendritic cells in murine epidermis are derived from bone marrow precursors. J Invest Dermatol 1984; 83 : 83-7.

18. Romani N, Tschachler E, Schuler G, et al. Morphological and phenotypical characterization of bone marrow-derived dendritic Thy-l positive epidermal cells of the mouse. J Invest Dermatol 1985; 85 : 91s-5s.

19. Bergstresser PR, Sullivan S, Streilein JW, Tigelaar RE. Origin and function of Thy $-1^{+}$ dendritic epidermal cells in mice. $J$ Invest Dermatol 1985 ; 85 : 85s-90s.

20. Romani N, Stingl G, Tschachler E, et al The Thy-l-bearing cell of murine epidermis : a distinctive leucocyte perhaps related to natural killer cells. J Exp Med 1985; 161 1368-83.

21. Caughman SW, Breathnach SM, Sharrow SO, Stephany DA, Katz SI. Culture and characterization of murine dendritic Thy-1+ epidermal cells. J Invest Dermatol 1986 ; 86 : 615 . 24.

22. Takashima A, Nixon-Fulton JL, Bergs tresser PR, Tigelaar RE (manuscrit en préparation)

23. Stingl G, Koning F, Yamada $\mathrm{H}$, et al Thy $-1^{+}$dendritic epidermal cells express $T 3$ antigen and the $\mathrm{T}$-cell receptor gamma chain Proc Natl Acad Sci USA 1987 ; 84 : 4586-90.

24. Kuziel WA, Takashima A, Bonyhadi $\mathbf{M}$ et al. Regulation of $T$-cell receptor gamma chain RNA expression in murine Thy-1 dendritic epidermal cells. Nature 1987; 328 263-6.

25. Koning F, Stingl G, Yokoyama WM, et al. Identification of a T3-associated gammadelta $\mathrm{T}$ cell receptor on $\mathrm{Thy}_{\mathrm{-}} \mathrm{I}^{+}$dendritic epidermal cell lines. Science 1987 ; 236 : 834-6.

26. Rubenfeld MR, Silverstone AE, Knowles $\mathrm{DM}$, et al. Induction of lymphocyte differen tiation by epidermal cultures. J Invest Dermatol 1981; 77 : 221-4.

27. Chu AC, Patterson JAK, Goldstein G Berger CL, Takezaki S, Edelson RL. Thymopoietin-like substance in human skin. $J$ Invest Dermatol 1983 ; 81 : 194-7.

28. Granstein RD, Lowy A, Greene MI. Epidermal antigen-presenting cells in activation of suppression: identification of a new functional type of ultraviolet radiation-resistan epidermal cells. J Immunol 1984 ; 132 : 563-5.

29. Kupper TS, Ballard DW, Chua AO, et al. Human keratinocytes contain mRNA indistinguishable from monocyte interleukin 1 alpha and bêta mRNA. Keratinocyte epidermal cell-derived thymocyte activating factoris identical to interleukin 1. J Exp Med 1986;

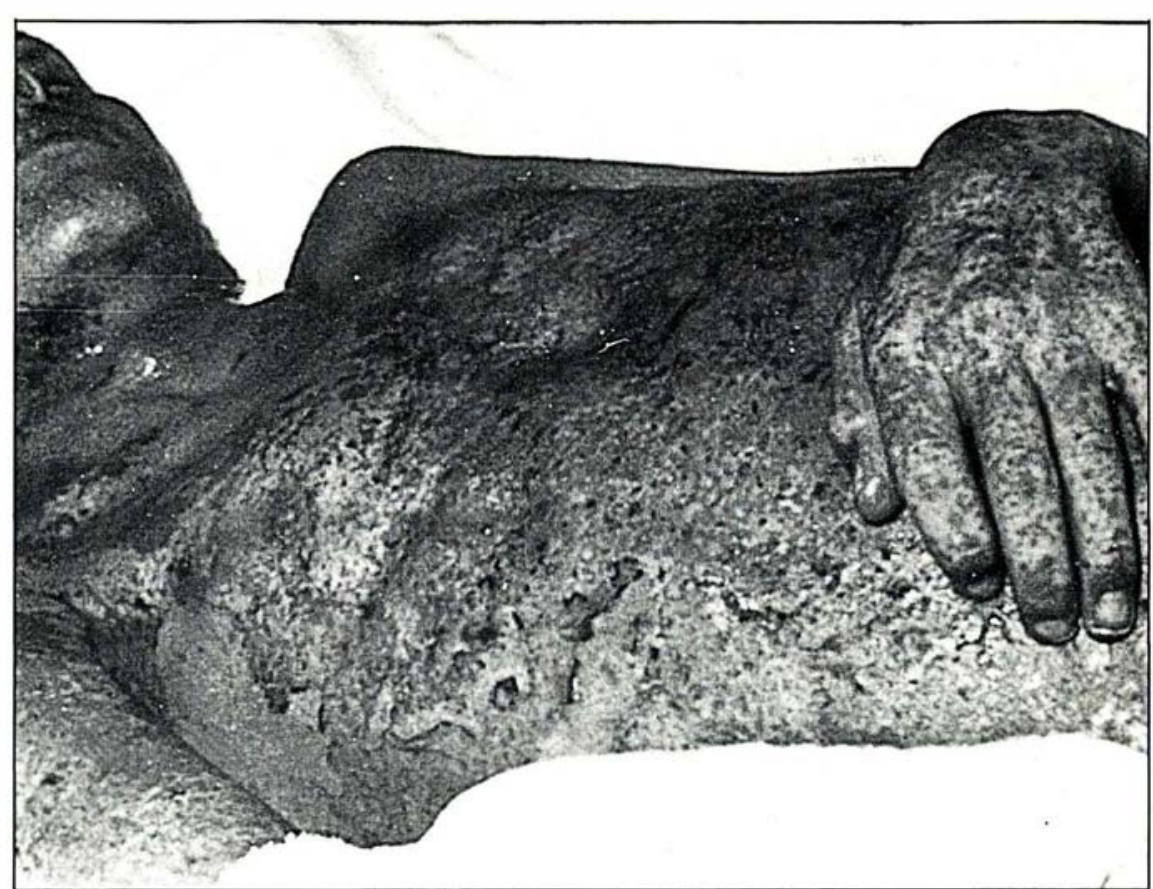

Figure 3. Aspect clinique de GVH aiguë sévère. GVH = graft versus hostdisease, réaction du greffon contre l'hôte. (Photo, service du Pr. Rochant).

keratinocyte derived $T$ cell growth factor [30] et une cytokine ayant les mêmes propriétés que l'interleukine 3 [31]. L'épiderme réunit donc tous les éléments nécessaires pour l'induction et l'amplification d'une réponse immunitaire dont la peau peut aussi secondairement être la cible.

\section{Cultures mixtes lympho-épidermiques}

La présentation de divers antigènes aux lymphocytes $T$ par les cellules de Langerhans a pu être étudiée in vitro à l'aide des cultures mixtes lymphoépidermiques (CMLE) qui représentent l'équivalent de cultures mixtes lymphocytaires unidirectionnelles dans lesquelles les cellules stimulantes sont des cellules épidermiques. Les cellules de Langerhans sont indispensables pour l'activation lymphocytaire et la génération de lymphocytes $T$ cytotoxiques $[32,33]$. L'intensité des réactions de prolifération obtenues en utilisant comme cellules stimulantes des cellules épi- dermiques est habituellement supérieure aux réponses induites par des cellules mononucléées sanguines autologues. Ceci a été démontré en réactions allogéniques [34, 35] et autologues modifiés par des virus ou des haptènes [35, 36]. La supériorité des cellules épidermiques est particulièrement nette en réaction primaire et pour de faibles quantités de cellules stimulantes, propriétés qui caractérisent les cellules de la lignée dendritique. Nous avons montré que les CMLE peuvent être positives entre deux frères ou sœurs HLAidentiques non réactifs en culture mixte lymphocytaire, ce qui peut être attribué soit à une meilleure présentation des antigènes mineurs d'histocompatibilité par les cellules de Langerhans, soit à des antigènes mineurs spécifiques des cellules épidermiques [37]. En effet, chez la souris, on a pu démontrer l'existence d'antigènes mineurs spécifiques de la peau, présents sur la membrane des cellules épidermiques et des fibroblastes dermiques mais non retrouvés sur les lymphocytes [38]. 


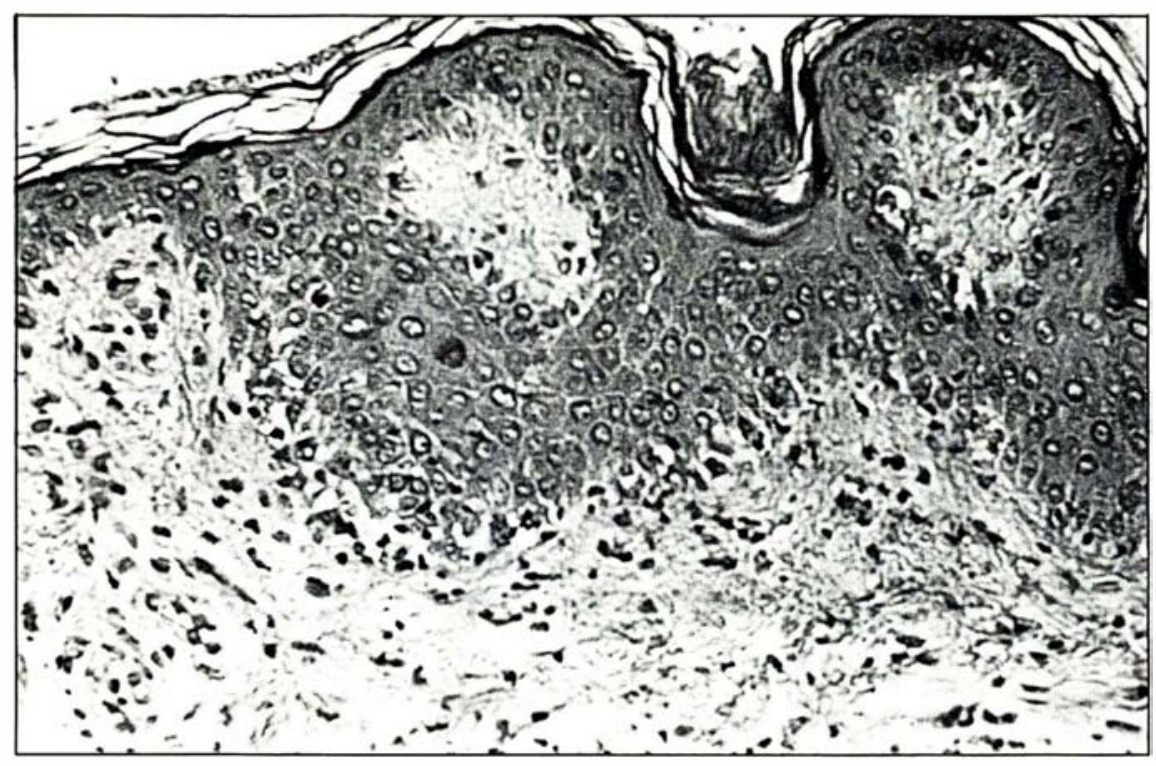

Figure 4. Aspect histologique de GVH aiguë. Cette coupe montre une dégénérescence vacuolaire des cellules basales de l'épiderme, un infiltrat lymphocytaire modéré dermique superficiel, et des images de satellite cell necrosis (contact cellulaire entre une cellule épidermique en voie de nécrose et une cellule mononucléée).

\section{Prédiction in vitro de la GVH}

Au cours des dernières années, la greffe de moelle allogénique humaine a constitué un progrès important dans le traitement de nombreuses maladies hématologiques. Cependant, la réaction du greffon contre l'hôte (graft-versus-host disease ou $\mathrm{GVH}$ ) reste la complication majeure de cette thérapeutique, bien que le donneur soit habituellement choisi parmi les frères et sœurs HLA-identiques du receveur. La

\footnotetext{
* Réaction de GVH chronique: phase de la maladie du greffon contre l'hôte de survenue tardive, plus de cent jours après la greffe. Cette réaction peut apparaître d'emblée ou, plus souvent, dans les suites d'une GVH aiguë. Son expression clinique et biologique ressemble à ce qui est observé au cours de certaines maladies auto-immunes.

* Lésions de type lichénien : lésions dermatologiques caractérisées cliniquement par des papules rouges ou violacées et histologiquement par un infiltrat lymphocytaire situé sous la membrane basale épidermique.

$\mathrm{m} / \mathrm{s} n^{\circ} 5$ vol. 4, mai 88
}

clone de lymphocytes cytotoxiques lysant spécifiquement l'antigène mineur Epa-1 [39]. On peut imaginer qu'une partie de la réaction observée en clinique humaine est induite par les cellules de Langerhans et dirigée contre des antigènes mineurs spécifiques de la peau du receveur.

Nous avons pu montrer que l'intensité de la prolifération obtenue en CMLE entre donneurs et receveurs de greffe de moelle est directement corrélée à l'incidence ultérieure de $\mathrm{GVH}$ aiguë et chronique[37, 40]. La CMLE constitue donc un véritable test prédictif de la réaction de $\mathrm{GVH}$ qui peut servir à choisir, au sein d'une fratrie, le meilleur donneur et à dépister les receveurs ayant un risque particulièrement élevé de GVH. D'autres auteurs ont rapporté des résultats similaires avec un modèle un peu différent. En effet, ils sensibilisent in vitro les lymphocytes du donneur avec les lymphocytes irradiés du receveur, avant de les mettre en co-culture avec une biopsie de peau du receveur. Les modifications histologiques constatées au niveau de la biopsie sont corrélées à l'incidence ultérieure de GVH aiguë[41]. Ces résultats pourraient avoir des conséquences importantes pour le traitement des patients recevant une greffe de moelle, en permettant de mieux déterminer les indications des déplétions de moelle. réaction de GVH prédomine niveau de certains organes cibles: l'épithélium intestinal, les canalicules biliaires et surtout la peau. Au niveau cutané, les lésions de GVH aiguë varient du simple érythème au grand décollement épidermique (figure 3) et les examens histologiques mettent en évidence une nécrose des cellules basales de l'épiderme avec parfois des images de satellite cell necrosis caractéristiques : contact cellulaire entre une cellule épidermique en voie de nécrose et une cellule mononucléée (figure 4). La peau constitue également la cible principale de la réaction de GVH chronique $^{*}$, avec des lésions de type lichénien ou sclérodermiforme ${ }^{* *}$. Chez la souris, il a été bien démontré que des réactions de $\mathrm{GVH}$ aiguè pouvaient être induites par l'activation de lymphocytes $T$ matures du greffon réagissant contre des antigènes mineurs d'histocompabilité du receveur. Il a été possible d'induire des lésions cliniques et histologiques de GVH en injectant par voie intra-dermique un

\section{Conclusion}

L'épiderme et ses analogies avec le thymus suscitent actuellement un grand intérêt. La découverte des propriétés particulières des cellules de Langerhans et d'une nouvelle population de lymphocytes $T$ très immatures au sein de l'épiderme ont fait récemment l'objet d'un grand nombre de travaux. Cependant, de nombreuses inconnues persistent, en particulier concernant le rôle et la signification fonctionnelle de ces cellules. On peut espérer que ces progrès permettront une meilleure connaissance des fonctions.immunologiques de la peau ainsi que de la physiopathologie de nombreuses maladies dermatologiques, en particulier les lymphomes cutanés, l'eczéma de contact et les réactions lichénoïdes 


\section{RÉFÉRENCES}

30. Kupper TS, Coleman DL, McGuire J, Goldminz D, Horowitz MC. Keratinocytederived T-cell growth factor. A T-cell growth factor functionally distinct from interleukin 2. Proc Natl Acad Sci USA 1986; $83: 4451-5$.

31. Luger TA, Wirth U, Kock A. Epidermal cells synthesize a cytokine with interleukin 3 . like properties. J Immunol, 1985 ; 134 : 915-9.

32. Stingl G, Katz SI, Clement L, Green I Shevach EM. Immunologic functions of Iabearing epidermal Langerhans cells. J Immunol $1978 ; 121: 2005-13$.

33. Faure M, Frappaz A, Schmitt D, DezutterDambuyant C, Thivolet J. Role of HLA-DR bearing Langerhans and epidermal indeterminate cells in the in vitro generation of alloreactive cytotoxic $T$ cells in man. Cell Immunol 1984; $83: 271-9$.

34. Sontheimer RD. The mixed epidermal cell-lymphocyte reaction. Human epidermal cells elicit a greater allogeneic lymphocyte response than do autologous peripheral blood lymphoid cells. J Immunol 1983 ; 130 : 2612-4

35. Bagot M, Heslan M, Roujeau JC, Lebon P, Lévy JP. Human epidermal cells are more potent than peripheral blood mononuclear cells for the detection of weak allogeneic or viral-specific primary responses in vitro. Cell Immunol $1985 ; 94: 215-24$

36. Braathen LR, Thorsby E. Human epidermal Langerhans cells are more potent than blood monocyres in inducing some antigenspecific T cell responses. $\mathrm{Br} J$ Dermatol 1983 $108: 139-46$.

37. Bagot M, Cordonnier C, Tilkin AF, et al A possible predictive test for graft-versus-host disease in bone marrow graft recipients : the mixed epidermal cell-lymphocyte reaction. Transplantation 1986; 41 : 316-9.

38. Steinmuller D. Tissue-specific and tissuerestricted histocompatibility antigens. Immunol Today $1984 ; 5$ : 234-40.

39. Tyler JD, Galli SJ, Snider ME, Dvorak AM, Steinmuller D. Cloned Lyt $-2^{+}$cytolytic $T$ lymphocytes destroy allogeneic tissue in vivo. J Exp Med 1984; 159 : 234-43

40. Bagot $M$, Cordonnier C, Vernant JP Dubertret L, Rochant H, Lévy JP. Mixed epidermal cell-lymphocyte reaction in predic tion of acute graft-versus-host disease in bone marrow recipients. J Nat Cancer Ins $1986 ; 76: 1317-9$

41. Vogelsang GB, Hess AD, Berkman AW et al. An in vitro predictive test for graftversus-host disease in patients with genotypic HLA-identical bone marrow transplants. $N$ Engl J Med 1985 ; 313 : 645-50.

\section{Summary}

Among the past few years, several advances have been performed showing that the epidermis is not only a target for immune-mediated injuries, but also functions as an immunologic organ. Langerhans cells are bone marrow-derived and represent 3 to $5 \%$ of epidermal cells. They are characterized ultrastructurally by a distinctive intracytoplasmic organelle known as Birbeck granule, which is probably involved in endocytosis phenomenon and by their reactivity with anti-T6 monoclonal antibodies. Langerhans cells are the only epidermal cells that express MHC-Class II antigens and are related to dendritic cells. They play a major role in contact hypersensitivity induction and skin graft rejection. Keratinocytes represent the majority of epidermal cells. They normally express only MHC Class-I antigens but may be induced to synthesize Class-II antigens in several pathologic conditions with dermal lymphoid infiltration or after gamma interferon exposure. They produce soluble factors which can stimulate lymphocyte proliferation and differentiation. Recently, a new dendritic cell type has been identified in murine epidermis. These cells are bone marrow-derived, $\mathrm{Ia}^{-}$, express large amounts of Thy-l antigen and represent 0.8 to $2.7 \%$ of epidermal cells. Their phenotype is $\mathrm{Ly}-5^{+}$, a sialo$\mathrm{G} \mathrm{M} \mathrm{I}^{+}, \mathrm{L}$ y t $-\mathrm{1}^{-}, \mathrm{L}$ y t $-2^{-}$, L3T4-, sIg-. Clones and cell lines derived from these cells express the T3 antigen and a gamma-delta $T$. cell receptor complex. Epidermal cells induce alloreactive and hapten-specific lymphoid proliferation and cytotoxic T-cell generation, which have been studied in vitro in the mixed epidermal celllymphocyte reaction (MECLR). MECLR can be positive between HLA-identical bone marrow donors and recipients and the intensity of the proliferation is correlated with later incidence of graft-versus-host disease. 